\title{
Prosthodontic Rehabilitation with Onlay Removable Partial Denture: a Case Report
}

\author{
Chang Wei Zhi ${ }^{1}$, Ho Ting Khee ${ }^{2 *}$ \\ 1. Faculty of Dentistry, Universiti Kebangsaan Malaysia, Jalan Raja Muda Abdul Aziz, 50300 Kuala Lumpur, Malaysia \\ 2. Department of Prosthodontics, Faculty of Dentistry, Universiti Kebangsaan Malaysia, Jalan Raja Muda \\ Abdul Aziz, 50300 Kuala Lumpur, Malaysia. \\ *E-mail: tingkhee.ho@ukm.edu.my
}

\begin{abstract}
Restoring occlusal plane and occlusal vertical dimension (OVD) in patient with existing indirect restorations who must wear removable prostheses may be a challenge to the dental operator. Onlay removable partial dentures (RPD) are used to re-establish the occlusion in conjunction to replace missing teeth without having to remove the existing indirect restoration. This case report described prosthetic rehabilitation with onlay RPD in patients who were partially edentulous and has reduced OVD. The treatment involved a set of provisional RPD to re-establish the OVD, as well as to evaluate the function, esthetic and speech of the patient, followed by definitive onlay RPD. The mandibular onlay RPD was used to provide stable occlusion and to correct the uneven occlusal surface on the abutment teeth. This treatment able to improve patient's chewing function through a simple removable prosthesis and conservative on the existing restorations.
\end{abstract}

Keywords: dental prostheses, overlay denture, partially edentulous, vertical dimension

\section{Introduction}

Overdenture refers to any removable partial denture (RPD) that covers or rests on one or more remaining natural teeth, their roots or dental implant. ${ }^{1}$ On the other hand, onlay RPD is a subset of overdenture where RPD has part of their components such as onlay covering the occlusal surface of the abutment teeth. ${ }^{2}$ It can be used to restore facial aesthetic, masticatory function and speech via conservative modification of existing dentition and particularly applicable to increase vertical dimension of occlusion and to improve an uneven occlusal plane. Onlay RPD is most often used as interim prostheses for patient with reduced occlusal vertical dimension (OVD) prior to fixed full mouth rehabilitation or prior to combined treatment of fixed and removable prostheses. ${ }^{3}$ Besides, it can also be used as a definitive prosthesis to re-establish vertical dimension and achieve stable occlusion. ${ }^{2,} 4$ Some clinicians prefer to have fixed restorations in increasing OVD instead of removable prostheses due to predictable patient's comfort and adaptation. However, the efficacy, simplicity, reversibility, conservative and low cost of RPD has enhanced its use widely. Nevertheless, there is not much scientific evidence on onlay RPD being prescribed to patient with reduced OVD.

The loss of OVD is a possible consequence of tooth wear as well as loss of posterior support due to missing posterior teeth. A physiological tooth wear usually happened over a long period of time and can be preserved by dento-alveolar compensatory mechanism via extrusion of worn teeth and alveolar bone remodeling. ${ }^{5}$ However, pathological tooth wear will need intervention to prevent further loss of hard tissue and to restore the form and function of the dentition. Altered OVD can be accessed through several clinical examinations such as loss of posterior teeth, signs of tooth wear, phonetic evaluation, interocclusal distance and facial appearance of drooping commissures. ${ }^{4} \mathrm{~A}$ reduced OVD from tooth wear and loss of posterior support is always a challenge to the dental operator due to limited interocclusal space for restorations. Hence, one of the management to such problem includes increase in OVD and it should be approached with caution as it may result in harmful consequences such as hyperactivity of masticatory muscles, elevation in occlusal forces, bruxism and temporomandibular disorder. ${ }^{6}$

There are several approaches to gain interocclusal space as well as re-establish the vertical dimension. This includes the records of maxilla-mandibular relationship (MMR) in centric relation, where mandible is at its most superior and posterior position of glenoid fossa. By recording the difference between centric relation and maximal intercuspation, horizontal space obtained interincisally can be utilized for tooth restoration. ${ }^{6}$ Although 
adequate interocclusal clearance may exist in intercuspal position (ICP) or there may be marked deviation between ICP and retruded contact position (RCP), space can also be acquired via placement of restorative material in supraocclusion, subtractive tooth preparation and a planned overall increase in patient's OVD. ${ }^{7}$ The ideal OVD to be reestablished can be obtained by determining the rest vertical dimension (RVD) from MMR in centric relation and subtracting the physiological freeway space of 2 to $4 \mathrm{~mm}$ from it. Several studies also showed that fixed and removable prostheses are reliable to re-establish vertical dimension, to provide space for restorative materials, enhance aesthetic tooth display, and minimize the need for biologically invasive clinical surgery and elective endodontic treatments. ${ }^{8,9}$

This case report described prosthetic rehabilitation with onlay RPD in patient who was partially edentulous with multiple indirect fixed restorations, occlusal wear of lower teeth and has reduced OVD. The treatment involved a maxillary provisional RPD to reestablish the OVD, as well as to evaluate the function, esthetic and speech of the patient. This is then followed by definitive maxillary Co$\mathrm{Cr}$ and mandibular onlay RPD. Besides re-establishing the vertical dimension in conjunction to replacing missing teeth without the need to remove existing indirect restorations, the mandibular onlay RPD was used to provide stable occlusion and to correct the uneven occlusal surface on the abutment teeth. This treatment able to improve patient's chewing function through a simple removable prosthesis and conservative to the existing restorations.

\section{Case Report}

A 53-year-old Chinese female presented to the dental clinic in Faculty of Dentistry at National University of Malaysia (UKM) with chief complaint of unretentive upper denture and loss of multiple teeth which affect chewing function and esthetic appearance. She had two maxillary dentures before but fractured upon mastication and during cleaning respectively. Dental history also suggested multiple fixed restorations done by previous dentist. Medical history revealed mild iron-deficiency anemia. She took iron tablets as supplement and vitamin $\mathrm{C}$ to increase iron absorption in body as prescribed by her medical practitioner.

Upon extra-oral clinical examination, patient exhibited no signs or symptoms of temporomandibular disorder but with dropping commissures around the mouth (Figure 1). Intra-orally, patient presented with Kennedy Class II modification I for upper arch (Figure 2) and Kennedy Class II for lower arch (Figure 3), had a fair oral hygiene with no deep periodontal pocket indicating absence of active periodontal disease. Multiple restorations were noted, with fixed prostheses on tooth 35 , being restored with porcelain-fused-to-metal crown, tooth 44 restored with metal onlay and tooth 46 restored with full-metal crown. At RCP, the upper premolar was having light contact with the lower right premolar, and the mandible was translated anteriorly and vertically into maximum intercuspation. At ICP, the upper premolar was occluding on the onlay and crown on tooth 44 and 46 respectively. Patient also presented with reduced OVD due to loss of posterior teeth. In addition, mild attrition exposing dentine was also found on the over-erupted mandibular teeth, involving tooth 33 to 42 . Patient had an unstable occlusion where she tended to protrude the mandible to achieve maximum intercuspation (Figures $4-6$ ). With rest vertical dimension (RVD) when the mandible was at rest and OVD being measured at $65 \mathrm{~mm}$ and $60 \mathrm{~mm}$ respectively, there was an increase of freeway space of $5 \mathrm{~mm}$.
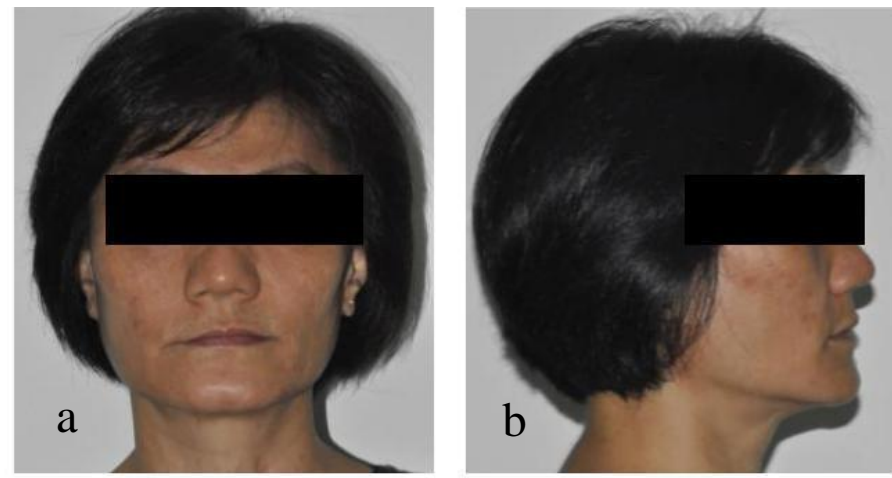

Figure 1. (a) Frontal view of patient with overclosure of mouth. (b) Right profile view showing drooping lips commissures and protruded chin.

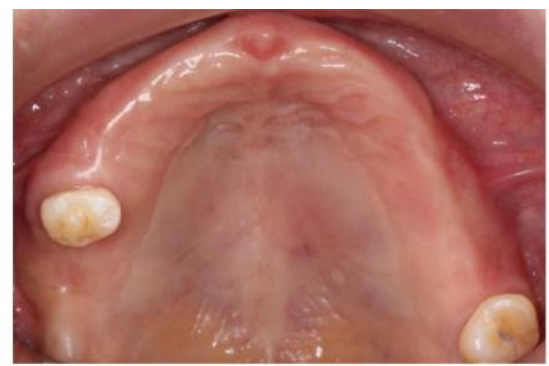

Figure 2. Preoperative occlusal view of maxillary arch demonstrating Kennedy Class II Modification I. Presence of tooth 15 and 28 .

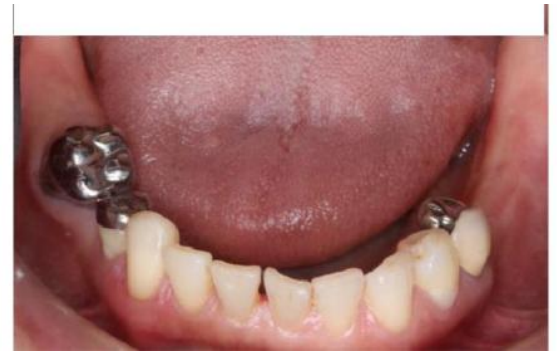

Figure 3. Preoperative occlusal view of mandibular arch demonstrating Kennedy Class II with presence of tooth 35 to 46 . Tooth 35 was restored with porcelain-fused-to-metal crown, 44 with metal onlay and 46 with full metal crown. Mild 
attrition was shown from tooth 33 to 42 , with slight overeruption of lower teeth.

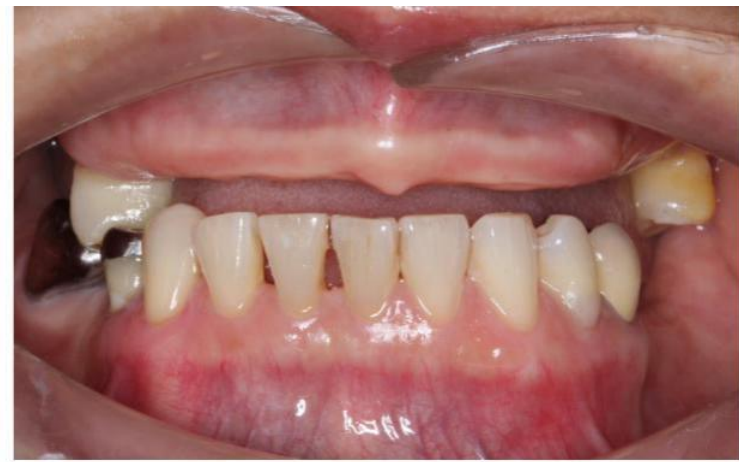

Figure 4. Preoperative frontal view of ICP. There is presence of limited vertical space between the upper alveolar ridge and lower teeth for removable prostheses.

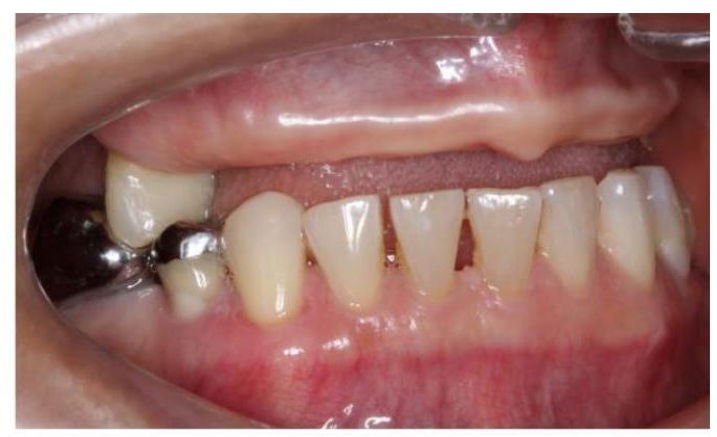

Figure 5. Right view of ICP. Tooth 15 is the only tooth occludes to the lower teeth.

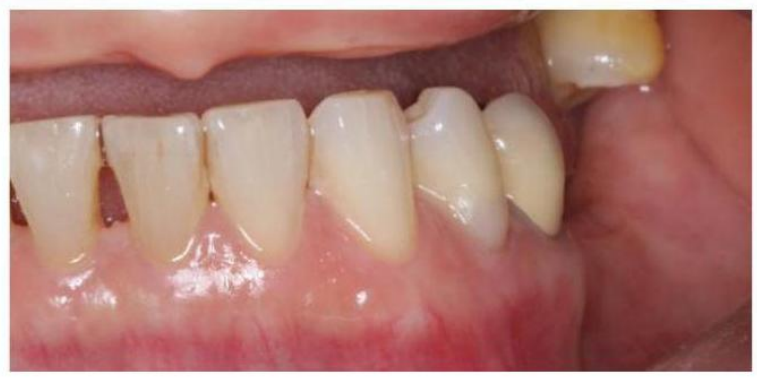

Figure 6. Left view of ICP. Tooth 28 is not in occlusion with the lower teeth.

The treatment plan was to restore the vertical height, facial appearance, mastication and phonetics. Treatment options were presented to the patient in the following order: (1) Re-construction of fixed crowns for mandibular posterior teeth at centric occlusion by aiming to restore the OVD and occlussal plane followed by maxillary RPD, (2) maxillary RPD at appropriate OVD and mandibular onlay RPD. Patient was fully informed of all risks, benefits and alternatives, and decided on treatment option \#2 as it was less destructive, less time consumed and less expensive.
Fabrication of provisional maxillary acrylic denture was done to re-establish the OVD and has been wore for 6 weeks to determine patient's adaptation and comfortability to the new OVD (Figures 7 - 9).The impressions were made with addition silicone polyvinyl siloxane (Aquasil Ultra, Dentsply International, York, PA, USA) to fabricate definitive casts for provisional denture. The new OVD was determined using upper baseplate with wax rim during the maxilla-mandibular (MMR) jaw registration stage and jaw record at centric relation. The orientation of upper occlusal plane was determined according to the ala-tragus line and interpupillary line. The anterior incisal level was determined based on patient's preference of $1 \mathrm{~mm}$ below upper lip line. The freeway space was reduced to $3 \mathrm{~mm}$ by re-established the OVD to $62 \mathrm{~mm}$ with RVD remained.

Wax setup of denture acrylic teeth was tried in to evaluate the OVD, occlusion, phonetic and appearance. The provisional maxillary denture was then processed and delivered. Denture hygiene care was given to patient and she was instructed to wear all day except night time to enhance adaptation to the increase vertical dimension. The patient was follow-up at one-, four- and six-week postoperative visits and minor adjustment to the denture was made to suit patient's comfortability. Upon examination, patient reported no muscle or temporomandibular joint tenderness, clicking nor deviation. In addition to satisfaction with the facial appearance, she was also comfortable and functioned well with the maxillary denture. However, the occlusal stability was not achieved as the abutment tooth 15 was not occluding to the mandibular fixed prostheses (Figure 8). This was one of the reasons why onlay RPD was planned for mandibular arch to achieve stable occlusion.

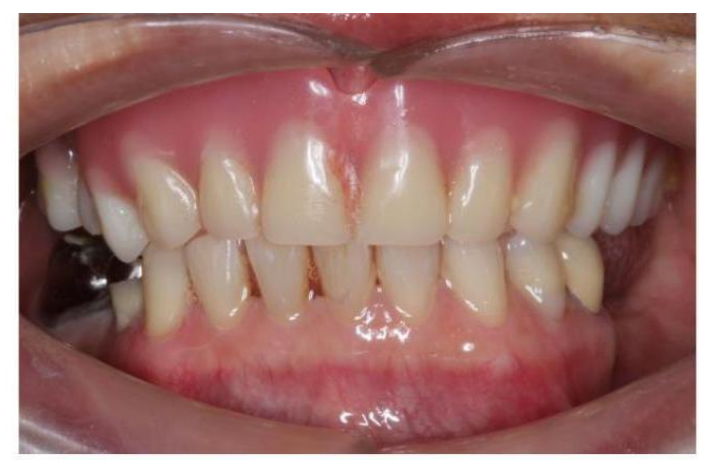

Figure 7. Frontal view of interim upper acrylic denture in centric occlusion 


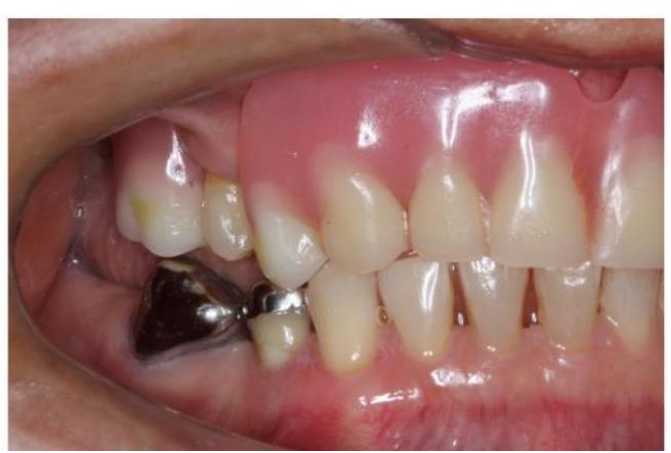

Figure 8. Right view of interim upper acrylic denture. Note that tooth 15 is not in occlusion with the lower 44 and 46.

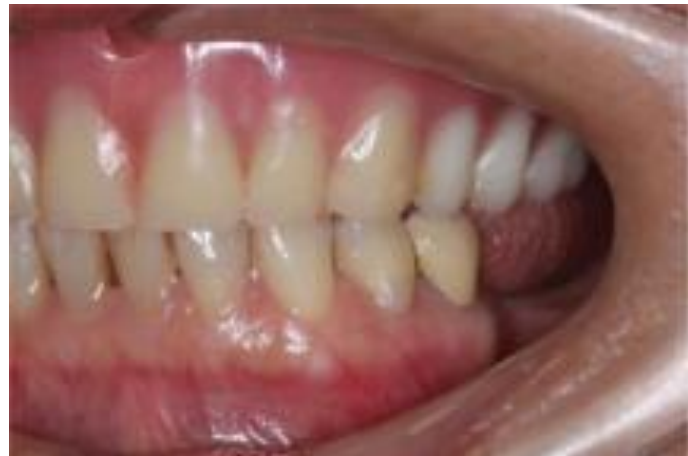

Figure 9. Left view of interim upper acrylic denture.

After six weeks of using provisional dentures without sign and symptom, definitive prostheses were made of Cobaltchrome $(\mathrm{Co}-\mathrm{Cr})$ at the same OVD and occlusion as of provisional denture. The maxillary $\mathrm{Co}-\mathrm{Cr}$ denture (Figure 10) and mandibular onlay $\mathrm{Co}-\mathrm{Cr}$ denture (Figure 11) at established OVD had achieved an even and stable occlusion (Figures 12 - 14). The onlay was seated on fixed prostheses of tooth 44 and 46 when onlay RPD was loaded in patient's mouth, and was in occlusion with upper tooth 15 (Figure 13). In this way, occlusal stability was attained in re-established OVD and patient can have better adaptation and comfort during functioning. The patient was followed up at one week, one month, and finally six months. She reported comfortable with the prostheses, able to perform mastication and speech effectively. Besides, she was satisfied with the facial appearance (Figures 15 and 16).

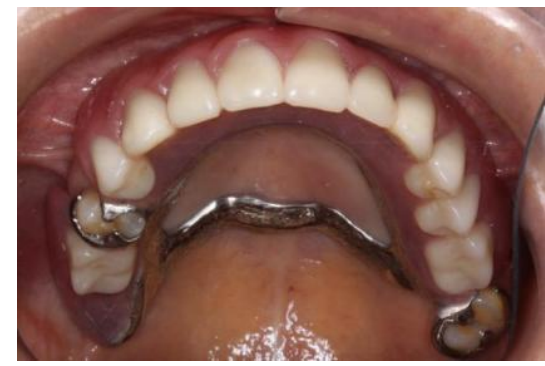

Figure 10. Postoperative occlusal view of maxillary arch restored with Cobalt-chrome $(\mathrm{Co}-\mathrm{Cr})$ denture.

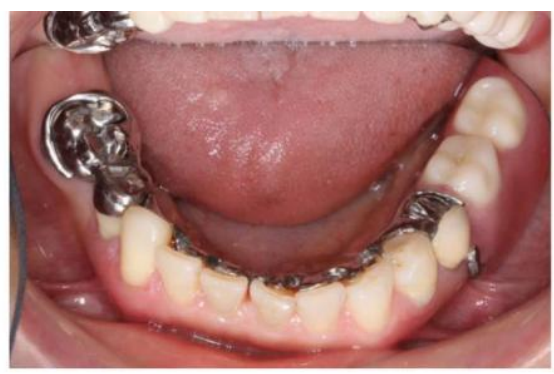

Figure 11. Postoperative occlusal view of mandibular arch restored with $\mathrm{Co}-\mathrm{Cr}$ onlay removable partial denture (RPD). The onlay was seating partially on full metal crown of 46 and metal onlay of 44 .

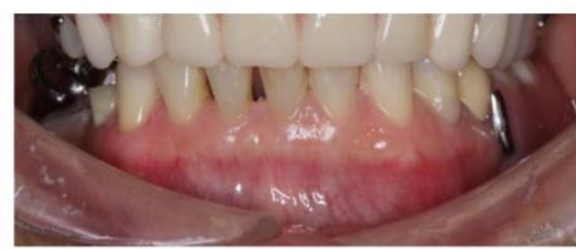

Figure 12. Postoperative ICP of upper $\mathrm{Co}$-Cr denture reestablishing the occlusal vertical dimension and lower onlay RPD achieving stable occlusion and even lower occlusal plane.

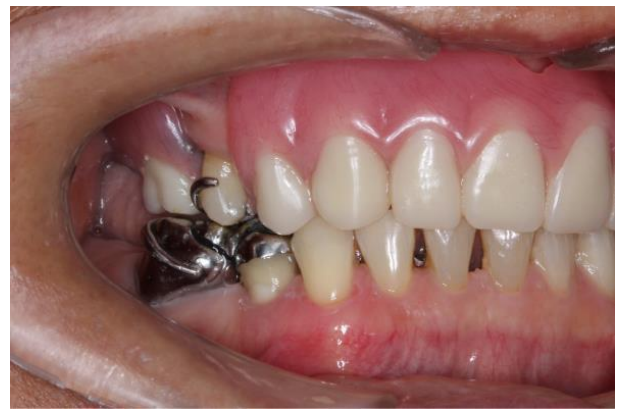

Figure 13. Right view of ICP, showing onlay RPD seating on occlusal surface of 44 and 46 to achieve an even lower occlusal plane. Besides, it is in contact with tooth 15 to achieve stable occlusion. 


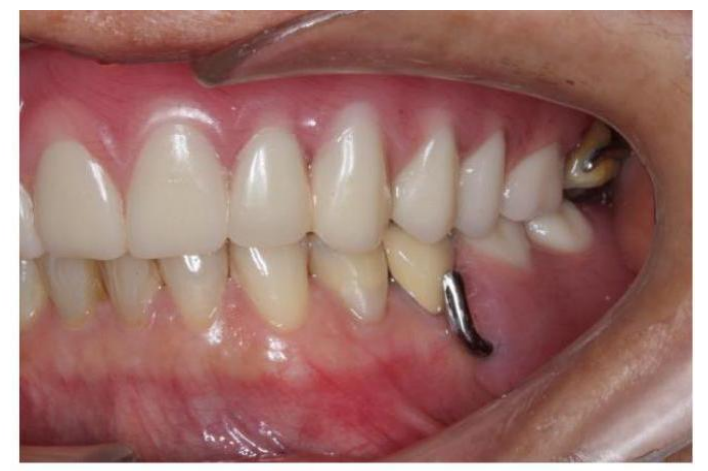

Figure 14. . Left view of ICP, with lower onlay RPD restoring the missing teeth and achieve stable occlusion.
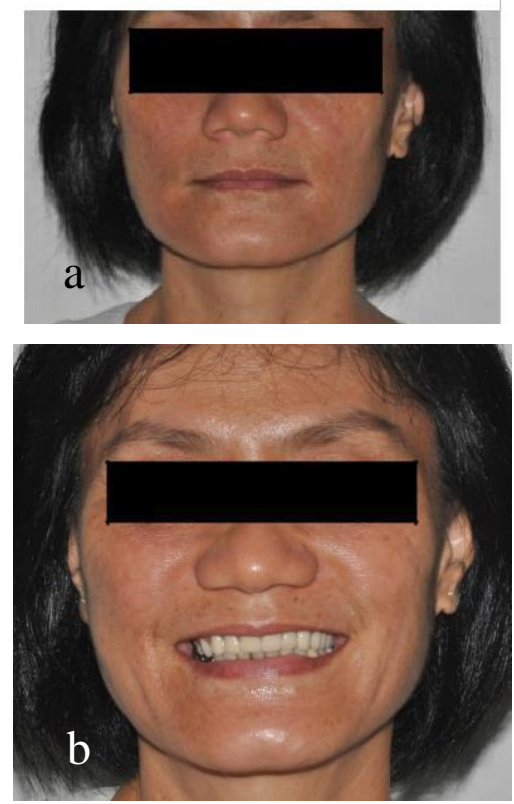

Figure 15. Postoperative frontal view of patient. (a) The reestablished vertical dimension of occlusion has eliminate the drooping of commissures. (b) A confident smile and aesthetic have been achieved.

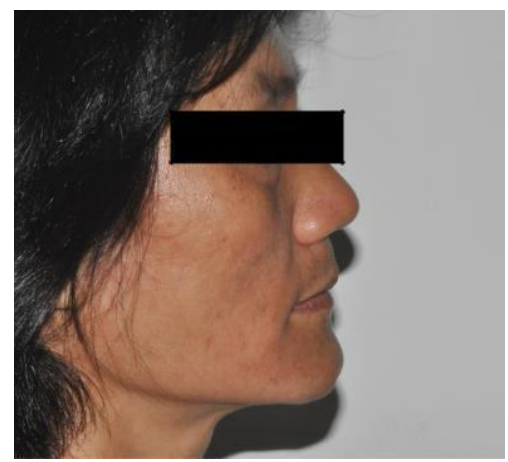

Figure 16. Postoperative right profile view of patient showing better nasolabial support, absence of commissural drooping and better overall facial aesthetic.

\section{Discussion}

Long term loss of posterior maxillary and mandibular teeth will result in unstable posterior support, uneven occlusal plane and tooth wear of mandibular teeth (tooth 33 to 42).This is due to deranged occlusion causing high occlusal load to the anterior teeth which lead to tooth attrition and aggravated by erosion, subsequently OVD will be reduced. In this case, $2 \mathrm{~mm}$ of OVD has been added to the maxillary $\mathrm{Co}-\mathrm{Cr}$ denture to re-establish the vertical dimension. A provisional period of 6 weeks with acrylic denture was given where patient reported no muscle or TMJ pain and feeling comfortable. According to a systematic review investigating the implications of increasing OVD, a permanent increase in vertical dimension from 1 to $5 \mathrm{~mm}$ is a safe and reliable procedure and the associated signs and symptoms are self-limiting with a tendency to resolve within 2 weeks. ${ }^{10}$ With such increase, there is sufficient interocclusal space for the placement of denture's acrylic teeth.

In addition, mandibular onlay RPD has been used as definitive prosthesis to achieve even occlusal plane and stable occlusion in a re-establised vertical height. ${ }^{11}$ The occlusal contacts allowed masticatory forces to be distributed in a simultaneous and homogenous way along the long axis of abutment. There was also a coordinated mandibular movement. In consideration of restorative procedures for the elderly patient that should be conservative, maintaining tooth structures as well as ensuring comfort and function, ${ }^{12}$ the onlay RPD was the best choice of treatment. Furthermore, the onlay RPD has been suggested to be simple, efficient, cost effective with the final outcome well pleasing to the patient. Patient was able to gradually accustomised to the newly adjusted vertical height with the use of onlay RPD and sufficient time was provided for remodeling of temporo-mandibular joint and occlusion. Besides aesthetic concerns, patient's clinical features of multiple teeth absence and posterior edentulous spaces were indeed a classical indication for onlay RPD.

The use of onlay RPD has eliminated the need of complicated treatment such as removing fixed prostheses, re-do fixed prostheses, surgical crown lengthening, orthodontically tilting of tooth, etc. The fixed restorations in this patient were also in satisfactory condition where their removal was not indicated. This has eventually reduced the risk of destructive fixed prostheses removal which includes pulpal exposure, tooth crack, tooth fracture and unnecessary tooth removal. In fact, a proper and thorough clinical examination and evaluation prior to any fixed restorations are crucial as they are irreversible. A good treatment plan should include assessment of patient's occlusion as a whole. If the fixed restorations (crowns and onlay) were at ideal vertical occlusal height, the fabrication of onlay RPD can be avoided and subsequently reduce the overall treatment cost.

Nevertheless, potential risks for framework component fracture, caries and periodontal disease if removable dentures are not well-maintained should be discussed with 
patient when comparing to fixed restorations. Therefore, regular recall and maintenance visits are essential for the longevity of abutments and long term success of maxillary $\mathrm{Co}-\mathrm{Cr}$ and mandibular onlay RPD. Patient should be reinforced with oral hygiene and denture hygiene especially with onlay RPD which act as plaque-retentive factor.

\section{Conclusions}

This case report describes the use of onlay RPD to achieve an even occlusal plane and stable occlusion in a re-established vertical dimension of occlusion. Provisional denture was used to temporarily increase the OVD and observe patient's adaptation before definitive treatment. This approach provided a safe and conservative route to meet the patient's requirement.

We successfully met the treatment goal of rehabilitation of chewing function and improved appearance. In patient with financial limitation for further fixed restorations, this may be a feasible or often the only treatment option. In a nutshell, patient with worn dentition and decreased OVD as well as missing teeth should be presented with this treatment option.

\section{Acknowledgment}

A special thanks to the dental technologist of UKM, Mr. Noorhaizad Ithnin for his expertise in providing laboratory support.

\section{References}

1. The glossary of prosthodontic terms. The Journal ofProsthetic Dentistry. 2005;94(1):58.

2. Patel MBB, S. A treatment protocol for restoring occlusa vertical dimension using an overlay removable partial denture as an alternative to extensive fixed restorations: A clinical report. The Open DentistryJournal. 2009;3:213-8.

3. Mehta SB, Banerji S, Millar BJ, Suarez-Feito JM. Current concepts on the management of tooth wear: part 3. Active restorative care 2: the management of generalised tooth wear.British Dental Journal. 2012;212(3):121-7.

4. Sato S, Hotta TH, Pedrazzi V. Removable occlusal overlay splint in the management of tooth wear: a clinical report. The Journal of Prosthetic Dentistry. 2000;83(4):392-5.

5. Berry DC, Poole DF. Attrition: possible mechanisms of compensation. J Oral Rehabil. 1976;3(3):201-6.

6. Abduo J, Lyons K. Clinical considerations for increasing occlusal vertical dimension: a review. Australian DentalJournal. 2012;57(1):2-10.

7. Mehta SB, Francis S, Banerji S. A Guided, Conservative Approach for the Management of Localized Mandibular Anterior Tooth Wear. Dent Update. 2016;43(2):106-8, 10-2.

8. Song MY, Park JM, Park EJ. Full mouth rehabilitation of the patient with severely worn dentition: a case report. JAdv Prosthodont. 2010;2(3):106-10.

9. Ergun G, Yucel AS. Full-mouth rehabilitation of a patient with severe deep bite: A clinical report. Journal ofProsthodontics. 2014;23(5):406-11.

10. Abduo J. Safety of increasing vertical dimension of occlusion: a systematic review. QuintessenceInternational. 2012;43(5):369-80.

11. Bataglion C, Hotta T, Matsumoto W, Ruellas C. Reestablishment of occlusion through overlay removable partial dentures: A case report. British Dental Journal. 2012;23(2):172-4.

12. Guttal S, Patil NP. Cast titanium overlay denture for a geriatric patient with a reduced vertical dimension. Gerodontology. 2005;22(4):242-5. 